\title{
Agressividade tributária: um survey da literatura
}

\section{Resumo}

Objetivo e Método: o objetivo deste artigo é revisar os estudos internacionais e no Brasil sobre a agressividade tributária, esta entendida como o comportamento de tentar evitar ou minimizar o ônus tributário explícito para a corporação. Enquanto tema de pesquisa acadêmica, o tax aggressiveness ou tax avoidance revela-se em um tópico diversificado e vasto, embora relativamente recente.

Resultados e Contribuições: Entre as perguntas de pesquisa que nessa temática se desenvolvem, destaquem-se a identificação de quais seriam os determinantes da agressividade tributária da firma, apreciando: (i) características das firmas; (ii) atributos ambientais; (iii) restrições de gatekeepers; e (iv) incentivos para as firmas. Revisam-se os determinantes da agressividade tributária dos gestores e a influência da governança e estrutura de controle. Adicionalmente, identificam-se as potenciais consequências econômico-financeiras da agressividade tributária para as firmas e as proxies empíricas existentes para mensurar a agressividade tributária. $\mathrm{O}$ estudo se encerra com a apresentação de oportunidades futuras de pesquisa na temática. Palavras Chave: Agressividade Tributária; Planejamento Tributário; Tax Avoidance.

\section{Antonio Lopo Martinez}

Doutor em Controladoria e Contabilidade pela Universidade de São Paulo (USP) e Professor Associado na Fucape Business School. Contato: Av. Fernando Ferrari, 1358. Bairro Boa Vista. Vitória (ES).

CEP.: 29075-505.

E-mail: lopo@fucape.br 


\section{Introdução}

Existe, na atualidade, um crescente interesse nos temas associados à agressividade tributária e ao planejamento tributário, tendo a temática obtido ainda mais destaque nos últimos anos em face de uma combinação de fatores políticos, econômicos e tecnológicos, que têm impulsionado o foco do interesse público para as decisões corporativas, em especial aquelas relativas a tributação (Guenther, Matsunaga \& Williams, 2017; Wilde \& Wilson, 2017). Como reflexo desse crescente interesse, destaquem-se as novas regras do Base Erosion and Profit Shifting (Beps), estruturadas com o claro propósito de minimizar a possibilidade de planejamento tributário internacional para empresas multinacionais («BEPS Actions OECD», 2017).

Inegavelmente, o planejamento tributário é crucial para qualquer corporação na tentativa de buscar redução de custos, tornando-se uma parte importante das decisões estratégicas (Klassen, Lisowsky \& Mescall, 2016). Por sua vez, as autoridades fiscais têm demandado uma maior transparência das atividades tributárias das empresas, que vão além da divulgação de informações rotineiras a padrões de relatórios por país, country by country report (Towery, 2017).

Aprimoramentos em normas de contabilidade financeira que criam trade-offs entre os relatórios financeiros e os incentivos fiscais (De Simone, 2016), as exigências do público em geral contra as práticas de planejamento tributário abusivo e a agressividade tributária por grupos ativistas (Dyreng, Hoopes \& Wilde, 2016) e a imprensa especializada tem colocado as questões tributárias no centro das atenções (Davis, Guenther, Krull \& Williams, 2016). Desse modo, a pesquisa acadêmica sobre agressividade fiscal corporativa tem crescido em sintonia com esse interesse público, beneficiando de novas fontes de dados, melhorias em medidas de prevenção a planejamentos tributários abusivos e aprimoramento de técnicas econométricas (Bird \& Karolyi, 2017).

O instrumental de Scholes-Wolfson foi marcante, sendo visto como um modelo para examinar as decisões empresariais de planejamento tributário (Shackelford \& Shevlin, 2001). Até meados da década de 1980, estudos jurídicos e análise econômica de política fiscal dominaram a pesquisa tributária. Somente com o quadro de Scholes-Wolfson (SW), passou-se a enfatizar a importância de considerar "todas as partes, todos os tributos e todos os custos" em avaliar as decisões de gestão fiscal (Scholes, Wolfson, Erickson, Hanlon, Maydew \& Shelvon, 2015). O desenvolvimento deste quadro levou a um aumento significativo nas pesquisas positivas em contabilidade tributária, na década de 1990, que, em grande parte, explora os trade-offs básicos entre custos tributários e não tributários (Austin \& Wilson, 2017).

$\mathrm{O}$ conhecimento da literatura internacional no tocante à agressividade tributária se expandiu consideravelmente em anos recentes, embora como nós propomos analisar, lacunas relevantes ainda permanecem (Hanlon \& Heitzman, 2010). No Brasil, as pesquisas na área apenas começam a se desenvolver, restando muitos pontos obscuros a serem investigados, em particular em face das especificidades da realidade brasileira (Araújo \& Leite Filho, 2017). Destaque-se que podemos constatar a rápida mudança recente em empresas no que se refere a política e ambientes de informação contábil, mudando os determinantes e consequências da agressividade fiscal (Wilde \& Wilson, 2017).

Nas seções que seguem, discutimos as descobertas e oportunidades de pesquisas na área da agressividade tributária no contexto da literatura contábil. Almeja-se descrever um quadro específico, examinando a variação transversal na temática Agressividade Tributária.

Em especial, pretendem-se discutir as oportunidades de pesquisa no Brasil no tocante aos seguintes elementos: i) determinantes da agressividade corporativa; ii) problemas de agência subjacentes no cenário de agressividade fiscal, incluindo o papel da governança, estrutura de propriedade e incentivos gerenciais; iii) o papel de gerentes e atributos pessoais identificáveis dos gestores sobre a agressividade tributária das empresas; e iv) consequências econômicas e financeiras para as empresas que adotam determinadas posturas em termos de agressividade tributária. 


\section{Conceituando Agressividade Tributária}

Por uma questão didática, parece necessário definir os conceitos-chaves a serem utilizados neste survey. Na verdade, hoje em dia, termos como "Evasão Fiscal", "Planejamento Tributário", "Planejamento Tributário Agressivo" e "Planejamento Tributário Abusivo" são amplamente utilizados, mas muitas vezes indefinidos (Dourado, 2017).

No contexto da legislação brasileira, não se definem Planejamento Tributário, Planejamento Tributário Abusivo ou Planejamento Tributário Agressivo. Também não encontraremos esclarecimentos sobre essas questões em regulamentos administrativos (Schoueri \& Galendi Júnior, 2017).

Portanto, inevitavelmente, surge a dúvida do que é válido fazer para reduzir, postergar ou afastar a incidência de tributos ("tax avoidance"). Pesquisas internacionais dizem que responder a esta pergunta não é tarefa fácil (Lietz, 2013). No Brasil, esta dificuldade é agravada pelo fato de as figuras jurídicas formalmente apontadas para realizar tal balizamento - tais como "simulação", "fraude à lei", entre outras - na prática, são aplicadas de forma confusa, ou, simplesmente, não são aplicadas (Martinez \& Coelho, 2016).

O fato é que existe uma pressão atual para enfrentar o comportamento fiscal abusivo, em função das crescentes demandas do Governo por receitas tributárias. Embora a solução no direito nacional compatível com o Código Tributário Nacional não esteja ainda plenamente regulamentada em norma antielesiva de caráter geral, esta situação não significa que as autoridades tributárias não conseguiram dispor de instrumentos para combater a evasão fiscal e o planejamento fiscal abusivo.

O Planejamento Tributário (ou tax avoidance) geralmente é visto como benéfico para a empresa e seus acionistas desde que o planejamento dos custos tributários implique maiores fluxos de caixa e lucro líquido para a firma e residualmente para seus acionistas (Blouin, 2014). O Planejamento Tributário almeja a priori reduzir as obrigações fiscais, aproveitando as concessões legítimas e as isenções previstas na lei tributária; envolve o processo de organização das operações empresariais de forma a que as obrigações fiscais sejam otimizadas no seu mínimo montante. Se dois métodos forem possíveis para alcançar um objetivo, seleciona-se um que resulte em menor obrigação tributária.

O Planejamento Tributário insere-se no contexto do planejamento global do negócio, visando reduzir os tributos explícitos e implícitos (França, Moraes \& Martinez, 2015). De acordo com o chamado quadro de Scholes-Wolfson, planejamento fiscal efetivo: i) considera as posições tributárias de todas as partes em um contrato (abordagem multilateral); ii) considera todos os impostos, tanto explícitos quanto implícitos; e iii) reconhece a relevância de todos os custos, tanto os custos tributários como não tributários.

As práticas de Planejamento Tributário podem conduzir a redução das obrigações tributárias. Entretanto, dependendo do grau de intensidade e legalidade em como essas práticas são adotadas define-se o seu grau de agressividade tributária, que se materializa, pragmaticamente, na magnitude de redução dos impostos explícitos.

Essa abordagem está em consonância com a definição assumida por Hanlon e Heitzman (2010), que salientam que as atividades reais favoráveis à redução de impostos e às ações que são, propositadamente, realizadas para evitar pagamentos de impostos e/ou benefícios fiscais colaboram para definir a agressividade fiscal. Destaque-se, que a priori, nessa definição não se faz distinção entre práticas fiscais claramente legais, juridicamente duvidosas ou "escalas cinza", ilegais e, de fato, fraudulentas.

A entidade mais agressiva tributariamente, ao realizar uma transação, assegura-se de que é utilizada em cada oportunidade a opção que permite minimizar os impostos. Quando opera em regimes onde a lei fiscal é incerta ou aberta a interpretação, tenderá a assumir a posição fiscal que lhe seja mais favorável. Ao estruturar suas transações, sempre buscará as formas e alternativas que garantem a maior economia tributária. Ocorre que, em determinadas situações, essa conduta pode assumir posições juridicamente duvidosas, e que aos olhos da autoridade tributária podem ser interpretadas, inobstante legítimas na sua forma, como abusivas. 
A busca de uma maior agressividade tributária não implica, necessariamente, abusividade tributária, entretanto há o risco de que, na redução deliberada das obrigações tributárias explícitas, a entidade adote medidas que abusem da lei, fraus legis, ou que a substância legal esteja em contradição com a forma legal.

O Planejamento Tributário Abusivo não se confunde com a evasão fiscal, em face da nítida ilegalidade deste último. Entretanto, o primeiro é potencialmente sujeito a questionamento das autoridades tributárias por ter potencialmente se materializado mediante negócios anômalos ou elusão fiscal. Desse modo, ainda que nem todo planejamento fiscal agressivo é necessariamente abusivo, o prognóstico final dependerá da perspectiva daquele que aprecia a transação tributária e se ela encontra respaldo na legislação.

Essa questão é particularmente problemática no Brasil, onde o reconhecimento de um Planejamento Tributário Abusivo é controverso, dada uma dubiedade sobre a possibilidade de aplicação de uma interpretação econômica aos fatos tributários. A norma antielisiva introduzida na lei complementar brasileira faz referência a "transações dissimuladas".

A legislação brasileira atual, fundamentalmente, baseia-se na diferença entre a evasão fiscal (esquemas simulados) e o Planejamento Tributário legítimo. No entanto, nota-se que, em tribunais administrativos, exemplo Conselho Administrativo de Recurso Fiscais (Carf), julgam majoritariamente em uma abordagem de substância sobre a forma e usam doutrinas de "abuso de lei" para definir o critério de validade de casos fiscais (Schoueri \& Freitas, 2010).

O grau de Agressividade Tributária mensura o ímpeto do sujeito passivo em reduzir o seu ônus fiscal em tributos explícitos, sem fazer qualquer restrição quanto à legalidade dos procedimentos às normas vigentes. Por hipótese, na medida em que se aumenta o grau de Agressividade Tributária, incrementa-se o risco fiscal de que as transações na sua dimensão tributárias serão desconsideradas pela autoridade fiscal. O risco fiscal e a agressividade tributária, a despeito de serem conceito distintos, são grandezas correlacionadas positivamente por incrementarem no mesmo sentido.

Para ilustrar em um esquema pictográfico as possíveis classificações de Planejamento Tributário e do grau de Agressividade Tributária, inspirado em Lietz (2013), mas com adaptações conceituais, propõe-se a Figura 1 a seguir.

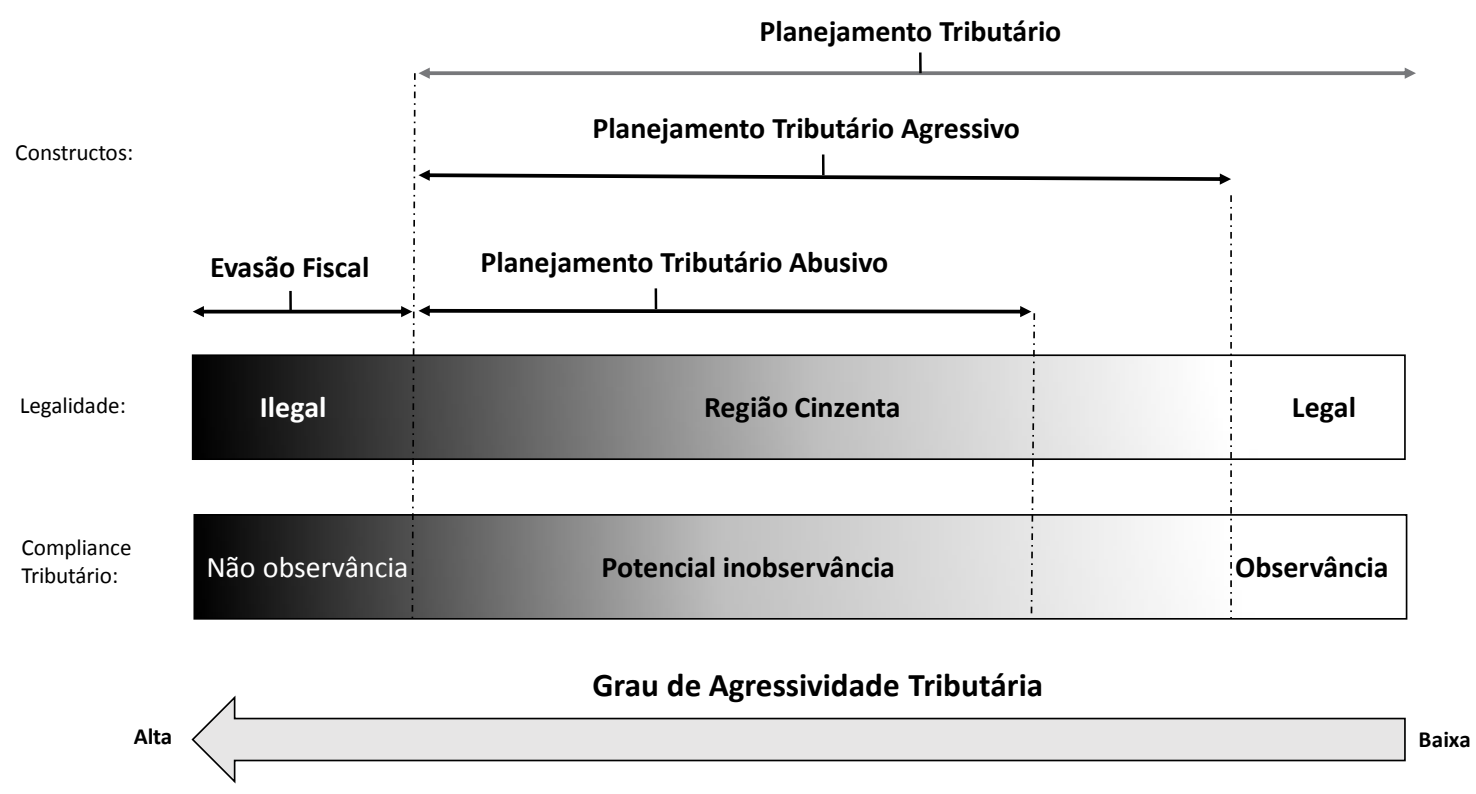

Figura 1. Planejamento Tributário e o Grau de Agressividade Tributária 


\section{Revisão da Literatura em Agressividade Tributária}

Existem ainda muitas oportunidades para pesquisas em torno do tema Agressividade Tributária (Hanlon \& Heitzman, 2010; Wilde \& Wilson, 2017). A área é muito fértil, e o interesse não se restringe apenas as autoridades tributárias, sendo também foco de atenção de público alvo muito abrangente, tais como gerentes, investidores e demais reguladores (Beng Wee Goh, Jimmy Lee, Chee Yeow Lim \& Shevlin, 2016).

As pesquisas no Brasil podem despertar também o interesse internacional em conhecer os fenômenos e objeto de estudo dentro do ambiente de países emergentes (Martinez \& Ramalho, 2014; Santana \& Rezende, 2016; Silva \& Rezende, 2017).

Apenas para destacar alguns pontos na literatura contábil tributária, este survey almeja desenvolver os seguintes tópicos gerais da agressividade tributária.

\subsection{Determinantes da agressividade tributária das firmas}

A literatura sobre a Agressividade Tributária se aproximou do Planejamento Tributário em uma visão focada na empresa, particularmente na ligação entre características das firmas e a agressividade fiscal em matéria de imposto sobre as sociedades (Armstrong, Blouin, Jagolinzer \& Larcker, 2015; Armstrong, Blouin \& Larcker, 2012). Sistematicamente, podem-se classificar nas seguintes categorias de determinantes propostas por Wilde \& Wilson (2017): (1) características das firmas; (2) atributos ambientais de onde as firmas atuam; (3) restrições dos gatekeepers; e (4) incentivos ao nível da firma para agressividade tributária.

\section{Características das firmas}

Pesquisas anteriores já examinaram várias características e particularidades das firmas e sua associação com a agressividade fiscal, incluindo tamanho da firma, planejamento de custos e operações internacionais (Desai \& Dharmapala, 2006, 2009; Dyreng, Hanlon \& Maydew, 2008).

Trabalhos mais recentes têm destacado a influência de algumas características da empresa no seu perfil agressividade fiscal, são elas: a qualidade do ambiente informacional da empresa (Gallemore \& Labro, 2015); a demora na publicação das demonstrações (Rodrigues, 2017); a predisposição para refazimento de demonstrações financeiras (Ramos, 2017); a eficiência dos mecanismos de controle interno (Martinez, Ribeiro \& Funchal, 2015; De Simone, Ege \& Stomberg, 2015; Bauer, 2016), a eficiência empresarial na utilização dos recursos (Paste Junior, 2017); as estratégias de negócios e os ciclos de vida das empresas (Higgins, Omer \& Phillips, 2015; Silva \& Rezende, 2017); as restrições financeiras as quais as empresas estão submetidas (Law \& Mills, 2015; Edwards, Schwab \& Shevlin, 2016; Da Silva \& Martinez, 2017; Richardson, Taylor \& Lanis, 2015); as transações com partes relacionas no exterior (Silva \& Martinez, 2017); e a utilização dos paraísos fiscais como mecanismo para redução da tributação explícita (Lee, 2017; Dyreng \& Lindsey, 2009; Dyreng, Lindsey, Markle \& Shackelford, 2015; Taylor \& Richardson, 2012). Esses estudos buscam identificar características das firmas que conduzem uma maior ou menor agressividade tributária.

Existem também pesquisas contemporâneas que associam Agressividade Tributária a características, tais quais a responsabilidade social corporativa e sua reputação (Davis et al., 2016; Hoi, Wu \& Zhang, 2013); marcas de consumo valioso (Watson, 2015); a conexões políticas (Brown, Drake \& Wellman, 2015; Mills, Nutter \& Schwab, 2013); uso de instrumentos financeiros derivados (Donohoe, 2015); fato de atuar em negócios diversificados (Zheng, 2017); e estar enfrentando dificuldades em planos de pensão (Chaudhry, Au Yong \& Veld, 2017). 


\section{Atributos ambientais}

Os atributos ambientais onde a firma atua se constituem em um determinante da agressividade tributária, tais como a concorrência de mercado do produto (Kubick, Lynch, Mayberry \& Omer, 2015), a concentração do clientes (Huang, Lobo, Wang, \& Xie, 2016), a atuação em mercados internacionais (De Simone, Klassen \& Seidman, 2017; Markle, 2016) e o conjunto de oportunidades de investimentos (McGuire, Omer \& Wilde, 2014) - todos esses são fatores ambientais que condicionam também a agressividade tributária.

Existem evidências de ligações entre a Agressividade Tributária e custos políticos potenciais (Mills et al., 2013) e os efeitos de repetidas anistias fiscais (Shevlin, Thornock \& Williams, 2017). Com a crescente concorrência global, a evolução tecnológica, as regras e a forma de evolução dinâmica do ambiente em que operam as empresas, há oportunidades para compreender o papel dessas condições na decisão sobre grau de Agressividade Tributária (Wilde \& Wilson, 2017).

O pano de fundo para grande parte da literatura de agressividade fiscal engloba considerações de relatórios contábeis e financeiros. Pesquisas anteriores oferecem evidência consistente de uma associação positiva entre os incentivos de mercado de capitais e o Planejamento Tributário (Lisowsky, 2010; McGuire, Omer \& Wilde, 2014; Wilson, 2009). Com as pressões do mercado de capitais sempre presentes e em um ambiente dinâmico de geração de relatórios financeiro, esta área provavelmente será promissora para futuras pesquisas (Dyreng, Hanlon, Maydew \& Thornock, 2017). Nesse sentido, merecem destaque os estudos que identificam os efeitos de adoção das normas contábeis internacionais e a Agressividade Tributária (De Simone, 2016).

\section{Restrições dos gatekeepers}

Como outra modalidade de pesquisa, destacam-se as que apreciam o papel dos gatekeepers no monitoramento da agressividade fiscal. Estudos documentaram que alguns gatekeepers, tais como redes corporativas (Brown \& Drake, 2013), contas de especialistas em indústria (McGuire, Omer \& Wang, 2012), fundos de hedge ativistas (Cheng, Huang, Yinghua Li \& Stanfield, 2012) e os investidores institucionais (Khan, Srinivasan \& Tan, 2017) facilitam ou inibem a agressividade tributária em certas configurações. Além disso, as evidências sugerem que a existência de departamentos fiscais internos e a contratação de assessores tributários especializados estão associados com maior Agressividade Tributária (Klassen et al., 2016).

Outras pesquisas destacam o papel dos gatekeepers em dissuadir a agressividade fiscal em contextos específicos, tais como com sindicatos (Chyz, Ching Leung, Zhen Li \& Meng Rui, 2013), cobertura de analistas de mercado de capitais (Allen, Francis, Wu, \& Zhao, 2016), investidores institucionais (Khurana \& Moser, 2013) e os empregados como próprios denunciantes (Wilde, 2017).

Qual o papel dos reguladores em limitar a agressividade tributária? Aqui, identificam-se tanto investigações teóricas (De Simone, Sansing \& Seidman, 2013) como empíricas (Bozanic, Hoopes, Thornock \& Williams, 2017). As pesquisas centram-se, predominantemente, no papel das autoridades fiscais na inibição da Agressividade Tributária (Hoopes, Mescall \& Pittman, 2012), a distância à autoridade fiscal da sede física do sujeito passivo (Kubick, Lockhart, Mills \& Robinson, 2017), a atuação dos reguladores financeiros, tais como o monitoramento da SEC, (Hope, Ma \& Thomas, 2013; Kubick et al., 2017) e o papel da transparência fiscal (Beck \& Lisowsky, 2014; Henry, Massel \& Towery, 2016). 


\section{Incentivos ao nível da firma}

Embora grande parte da investigação tenha centrado em incentivos monetários, ligados diretamente à economia tributária, trabalhos recentes têm analisado os custos reputacionais ligados à agressividade tributária. As preocupações com a reputação são um incentivo crítico, porém estudos com evidências empíricas ainda são limitados (Graham, Hanlon, Shevlin \& Shroff, 2014).

Em questionário aplicado a executivos internacionais, $69 \%$ deles classificam a reputação como importante, entretanto os incentivos de contabilidade financeira desempenham um papel mais relevante $84 \%$ das empresas de capital aberto respondem que a alta administração da empresa se importa com o GAAP ETR e 57\% das empresas abertas afirmam que aumentar o lucro por ação é um resultado importante de uma estratégia de Planejamento Tributário (Graham et al., 2014).

\subsection{Determinantes da agressividade tributária do gestor}

O estilo gerencial, peculiaridades e incentivos dos gestores influenciam também a Agressividade Tributária (Bertrand \& Schoar, 2003). Uma vez que os gerentes apresentam características únicas que os diferenciam em comportamento, é importante compreender quais os condicionantes da agressividade fiscal dos gestores.

As pesquisas consolidaram a noção de que os gerentes desempenham funções individuais significativas na definição do perfil de agressividade das empresas que gerenciam. Como regra geral, esses estudos usam modelos de regressão controlando determinados atributos dos gestores e seus efeitos na agressividade tributária.

As evidências desta linha de investigação apontam para associações significativas entre vários traços individuais dos gestores e a agressividade fiscal das empresas, tais como: a experiência militar do gestor (Law \& Mills, 2017); o narcisismo (Olsen \& Stekelberg, 2016); a orientação política e agressividade pessoal (Chyz, 2013); o gênero de seus executivos e dos membros do conselho de administração (Francis, Hasan, Qiang Wu, \& Meng Yan, 2014; Lanis, Richardson, \& Taylor, 2017); a religiosidade do gestor (Boone, Khurana \& Raman, 2013; Dyreng, Mayew \& Williams, 2012); suas habilidades e capacidade gerencial (Koester, Shevlin \& Wangerin, 2017); e o perfil de remuneração, pensão e incentivos compensatórios (Armstrong, Blouin \& Larcker, 2012; Chi, Huang \& Sanchez, 2017; Kubick \& Masli, 2016).

\subsection{Governança corporativa e agressividade tributária}

A literatura internacional é pródiga na identificação de aspectos de governança corporativa que podem interagir com a prática da Agressividade Tributária. Como mecanismos que eventualmente mitigam os seus efeitos, nesses aspectos notam-se vários estudos que relacionam governança corporativa, estrutura de propriedade e incentivos à compensação, todos interagindo para encorajar o gerente a tomar decisões ótimas de Planejamento Tributário (Badertscher, Katz \& Rego, 2013; Chan, Phyllis Lai Lan Mo \& Tanya Tang, 2016; Chen, Chen, Cheng \& Shevlin, 2010; Richardson, Wang \& Zhang, 2016; Salihu, Annuar \& Sheikh Obid, 2015).

No Brasil algumas pesquisas já relacionam aspectos de governança e agressividade tributária. Destaquem-se as pesquisas que apreciam os seguintes pontos: a relação entre empresas familiares e a Agressividade Tributária (Cabello \& Gaio, 2017; Martinez \& Ramalho, 2014); o controle por capital estrangeiro (Bis \& Martinez, 2017); a influência da estrutura de controle (Fonseca \& Martinez, 2017); o fato de a empresa ser controlada ou controladora (Martinez \& Dalfior, 2016); e as características gerais de governança corporativa, como a remuneração paga ao conselho executivo, a segregação entre Chairman e CEO e a independência e composição do Conselho de Administração (Gomes, 2016). 


\subsection{Consequências da agressividade tributária}

Qual é o efeito de curto, médio e longo prazo da Agressividade Tributária? Como efetivamente mensurar o trade off proposto no modelo de SW, no qual a maior agressividade tributária pode vir acompanhada de custos não tributários, destacando dentre outros, uma própria tributação implícita. Em pesquisas recentes internacionais, reforçam-se os efeitos da Agressividade Tributária no custo de capital próprio (Beng Wee Goh et al., 2016; Cook, Moser \& Omer, 2017), na estrutura de financiamento e maturidade da dívida (Kubick \& Lockhart, 2017) e na propensão a que ocorra um CEO turn-over (Chyz \& Gaertner, 2017).

Ao discutirmos as consequências da Agressividade Tributária, encontramos alguns trabalhos focalizados na realidade brasileira, dentre os quais destacaram-se os efeitos da agressividade tributária: no valor da firma (Santana \& Rezende, 2016), no custo de capital de terceiros (Martinez \& Martins, 2016; Martinez \& Silva, 2017), na relação de dependência entre os segmentos de governança corporativa da BM\&FBOVESPA e o retorno sobre ativo (Potin, Silva, Reina \& Sarlo Neto, 2016), no risco sistemático (Vello \& Martinez, 2014), na qualidade da previsão dos lucros por ação dos analistas (Carvalho, 2017) e no conservadorismo contábil (Vale \& Nakao, 2017).

Ainda nessa linha de identificação de consequências podem ser destacados os trabalhos no contexto do Brasil que avaliam os efeitos da Agressividade Tributária: no rating de emissões de dívidas das empresas (Miiller \& Martinez, 2016), na rentabilidade futura (Araújo \& Leite Filho, 2017; Reinders \& Martinez, 2016) e na remuneração exigida pelos auditores independentes (Martinez, Lessa \& Moraes, 2014).

\subsection{Proxies para mensurar agressividade tributária}

Após a pesquisa, Shackelford e Shevlin (2001) desenvolveram uma série de diferentes formas de mensurar o grau de agressividade fiscal. Muitos artigos surgiram desenvolvendo medidas, na sua maioria centrada nos tributos explícitos e independente se o resultado tributário estava ao abrigo de imposto juridicamente questionável ou muito agressivos (Brown, 2011; Hanlon \& Heitzman, 2010; Lisowsky, 2010; Wilson, 2009).

É crucial destacar que as medidas de agressividade fiscal estão centradas em apreciar a agressividade apenas nos tributos sobre o resultado contábil da entidade, que, no caso do Brasil, seriam fundamentalmente o Imposto de Renda Pessoa Jurídica e a Contribuição Social sobre o Lucro Líquido. Portanto, não se considera a agressividade em relação a qualquer outro tributo. Esse é um ponto que restringe fortemente a projeção das conclusões dessas pesquisas, especialmente na realidade brasileira, onde os tributos são os mais variados, diretos e indiretos, e a tributação sobre o resultado é apenas uma das modalidades, historicamente de menor relevância.

Muitos artigos utilizam o BTD (Book-Tax Diferences), medida pela diferença do lucro contábil e tributário, como uma proxy da Agressividade Tributaria. Nesta última linha, nota-se o trabalho que desenvolve uma medida da diferença permanente entre o lucro contábil e o tributário (Frank, Lynch \& Rego, 2009). O BTD como medida de agressividade tributária é viesada, pois o seu comportamento pode ser também definido pela propensão ao gerenciamento de resultados. Desse modo, é crucial controlar esse aspecto antes de poder inferir se a entidade está sendo efetivamente agressiva tributariamente (Ferreira, Martinez, Costa \& Passamani, 2012). 
As métricas de agressividade mais usuais são aquelas relacionadas com a taxa de tributação efetiva. A taxa tributária efetiva sobre o lucro contábil (Taxa_GaapETR) é apurada como Despesa Total com Tributos sobre o Lucro / Lucro antes dos Tributos (GAAP ETR), conforme proposto por Hanlon e Heitzman (2010), a qual é a métrica mais utilizada para indicar o grau de agressividade. Assim, uma baixa taxa GAAP ETR significa que uma empresa realiza Planejamento Tributário de forma mais agressiva do que as empresas com maior taxa GAAP ETR. Como variações dessa métrica, podem também ser apuradas as taxas efetiva total (ETRt) quanto às taxas efetiva corrente (ETRc), dependendo se considera a tributação diferida ou não (Guimarães, Macedo, \& Cruz, 2016). Merece também destaque uma possibilidade de ajuste proposto no ETR mais compatível à realidade brasileira. O Lucro antes dos tributos seria acrescido da reversão da despesa de JCP (lançada contra PL por determinação da CVM - Deliberação CVM n. ${ }^{\circ}$ 207/1996) e dos ajustes de equivalência patrimonial (Medeiros \& Costa, 2017).

Outra métrica relevante é a ETR long run (taxa de imposto efetiva de longo prazo). Esta métrica é atraente, comparada à taxa de imposto efetiva do GAAP tradicional divulgada nas demonstrações financeiras da empresa (Dyreng et al., 2008). Esta medida dinâmica de agressividade fiscal é oportuna para os pesquisadores interessados em documentar variação na atividade de agressividade fiscal, sem a necessidade de se concentrar unicamente em um conjunto limitado de transações, estáticas e de apenas um único período.

A Taxa_CashETR (taxa tributária efetiva de caixa) pode ser definida como Tributos sobre os Lucros Totais Pagos / Lucro antes dos Tributos. Temos desse modo uma ETR dos tributos efetivamente pagos (CashETR). A CashETR de uma empresa é a medida mais direta da carga fiscal de caixa de uma empresa, e o planejamento tributário que diminui a carga fiscal de caixa terá um impacto direto sobre a Cash ETR paga. A CashETR de curto prazo serve para capturar respostas oportunas às restrições financeiras existentes, pois há uma série de potenciais estratégias de Planejamento Tributário que as empresas podem implementar em um período de tempo relativamente curto, como, por exemplo: realizarem despesas de forma mais agressiva do que capitalizá-las; obterem vantagens de programas de incentivo fiscal; e envolverem-se em estratégias tempestivas que acelerem as deduções e que posterguem o reconhecimento de resultados (Da Silva \& Martinez, 2017).

Uma métrica genuinamente brasileira, e sem qualquer precedente para comparação internacional, a taxa tributária efetiva sobre o lucro adicionado (Taxa_DVA) é aqui definida como Carga Tributária da DVA / Valor Adicionado Total a Distribuir (Da Silva \& Martinez, 2017; Motta \& Martinez, 2015). Essa métrica é sui generis por dois motivos: primeiramente por partir da Demonstração de Valor Adicionado (DVA), diferente da maioria das métricas internacionais que utilizam exclusivamente a Demonstração de Resultado do Exercício (DRE); o segundo aspecto e mais relevante é o fato de a métrica captar uma medida de Agressividade Tributária mais abrangente do que a ETR. Essa medida mensura a agressividade em várias dimensões possíveis - todos os tributos, no âmbito federal, estadual e municipal (Fernandes, Martinez \& Nossa, 2013). Ante o exposto, verifica-se que, para uma análise geral da Agressividade Tributária, essa métrica oferece importantes vantagens sobre a ETR tradicional.

\section{Oportunidades Futuras de Pesquisa}

Se lançarmos um olhar interpretativo sobre a literatura contábil na área de Agressividade Tributária, notar-se-á uma explosão do número de artigos em torno da temática Planejamento Tributário ( $\operatorname{tax}$ avoidance) nos últimos anos, especialmente nos top journals de contabilidade (Wilde \& Wilson, 2017).

No tocante às pesquisas nessa temática no Brasil, surpreende-se com o fato de que elas são praticamente inexistentes. Existem poucas pesquisas publicadas na temática Agressividade Tributária, o que apenas por si cria inúmeras oportunidades. Investigadores interessados em apreciar as especificidades brasileiras poderão produzir conhecimento importante e relevante tanto a nível interno como internacional.

Na sequência indicamos algumas áreas que poderiam se beneficiar de estudos mais detalhados. 


\section{Relação entre o risco fiscal e a agressividade tributária}

Uma área em que a literatura se beneficiaria de uma análise mais profunda é a associação entre agressividade fiscal e risco fiscal. Fundamentalmente, a noção de que o risco fiscal expressa ainda tem pouco consenso na literatura, limitando nossa compreensão dos fatores subjacentes a esse risco? Pesquisas futuras podem tentar identificar as circunstâncias em que a Agressividade Tributária conduz a riscos adicionais. Desse modo podem fornecer informações sobre os fatores que deveriam influenciar as estratégias fiscais de uma empresa (Guenther et al., 2017). Qual a relação entre a Agressividade Tributária e a propensão a ser autuado por autoridade tributaria? No Brasil poder-se-ia tentar investigar qual o efeito das ações das auditorias tributárias na agressividade fiscal? (Bozanic et al., 2017; Hoopes et al., 2012; Kubick et al., 2017).

\section{Custos reputacionais das práticas agressivas tributárias}

Uma possível consequência negativa da Agressividade Tributária pode se manifestar em custos reputacionais. Nesse sentido, as empresas podem estar preocupadas em equilibrar o trade off das escolhas fiscais com as implicações reputacionais dessas estratégias (Austin \& Wilson, 2017). A prática de políticas de responsabilidade social, por exemplo, pode ser incompatível com as práticas de Agressividade Tributária, deste modo, mereceriam estudos mais detalhados no contexto do Brasil (Huseynov \& Klamm, 2012; Jones, Baker \& Lay, 2017; Laguir, Staglianò \& Elbaz, 2015). Os efeitos reputacionais ainda se tratam de um caminho inexplorado de investigações no Brasil. Haveria algum efeito reputacional no Brasil de entidade envolvidas em escândalos de fraude fiscal?

\section{Associação entre as escolhas contábeis de reporte e a agressividade tributária}

Como as decisões financeiras de reporte estão associadas com as estratégias tributarias? Inobstante variações em conformidade com o lucro contábil e lucro tributário, nota-se que, em várias ocasiões, as escolhas de contábeis influenciam os resultados tributários. Como essas escolhas interagem? Quais os incentivos que são mais pronunciados? Até que ponto seria possível, por exemplo, conviver com um suposto conservadorismo contábil e Agressividade Tributária? São complementares, ou substitutos?

A menor conformidade do lucro contábil ao lucro tributário assegura uma melhor ou pior qualidade do lucro contábil? Qualidade de lucros expressa nas suas mais variadas dimensões, isto é, baixo gerenciamento de resultados, persistência dos lucros, informatividade ao mercado, previsibilidade de lucro e conservadorismo contábil (Sundvik, 2017; Tang, 2015). A Agressividade Tributária está associada a uma agressividade no reporte contábil? (Lennox, Lisowsky \& Pittman, 2013).

\section{Aprofundar o estudo das consequências da agressividade tributária}

As pesquisas futuras devem assegurar uma melhor compreensão das consequências da agressividade fiscal das empresas. Gerentes racionais devem considerar as consequências da agressividade fiscal ao tomar suas decisões. Nesse sentido é pertinente identificar e enfatizar todas as possíveis consequências. A agressividade tributária tem consequências potenciais para gerentes, acionistas, credores e o governo. Qual o efeito desta prática no valor da empresa, na remuneração dos gestores, nos riscos do negócio, nos custos de capital de terceiros e próprio? (Cook et al., 2017; Kubick \& Lockhart, 2017; Seidman \& Stomberg, 2017). 


\section{Identificar novas métricas de agressividade tributária}

Os estudos internacionais enfatizam a agressividade sempre no que concerne aos tributos explícitos sobre o resultado contábil. Parece míope apreciar somente um tipo de pagamento dos tributos. Estudos devem buscar identificar métricas que avaliem a agressividade tributária nos diversos tributos, diferentes tipos e os tributos implícitos. Isso é particularmente crucial no contexto brasileiro, onde o sistema tributário é complexo e com variados tributos. Um caminho a ser trilhado e em que se pode, talvez, aumentar o conhecimento internacional, seriam pesquisas usando informações da DVA. Ainda relativamente à mensuração da agressividade fiscal, devem ser estimulados métodos alternativos, dentre os quais se destaca como um potencialmente interessante o BTD anormal, estimado a partir de um modelo econométrico, ajustado para os controles necessários (Brunozi Junior, 2016).

\section{Sujeito ativo, autoridade tributária e a agressividade tributária}

Qual o real efeito das ações da autoridade tributária para a agressividade tributária futura das empresas? Como os procedimentos fiscais impactam sobre a Agressividade Tributária em empresas individualmente, setores econômicos e grupos de contribuintes? Qual o efeito de programas de anistia fiscal sobre a Agressividade Tributária futura?

Recompensas positivas por obediência fiscal (Martinez, 2014), aplicação de multas, incremento de deveres instrumentais, uso intensivo de tecnologia de informação a serviço da administração tributária, todos esses fatores influenciam a Agressividade Tributária das empresas? Pesquisas devem ser estimuladas para permitir uma melhor compreensão de como as ações do sujeito ativo, ou particularmente da administração tributária, influenciam a agressividade tributária.

\section{Conclusão}

O presente artigo procurou reportar e promover uma análise da literatura em torno da Agressividade Tributária. Ao longo da investigação, revisaram-se estudos e reportaram-se resultados, que, progressivamente, firmaram conclusões. Deste modo as conclusões finais serão sucintas e pontuais.

A temática de Agressividade Tributária tem se demonstrado muito profícua com questões ainda não plenamente resolvidas que podem ser de interesse para formuladores de políticas tributárias, reguladores, incluindo aí as autoridades tributárias, diretores de empresas, investidores e pesquisadores acadêmicos. É inegável que as pesquisas nessa temática explodiram em anos recentes, como se pode notar em face da contemporaneidade dos artigos que foram revisados durante este survey.

Como tema emergente, nota-se presença recorrente nos top journals em Contabilidade, tais como, The Accounting Review, Journal of Accounting Research e Journal of Accounting and Economics. No Brasil, entretanto, as pesquisas são embrionárias, com poucos artigos publicados em periódicos nacionais, retratando a realidade local, sendo que a maioria das pesquisas revisadas ainda está em fase de exposição em eventos, ou ainda como dissertações e teses acadêmicas.

A temática enfrenta uma dificuldade conceitual para definir a agressividade fiscal, certamente motivado por uma ausência de definição jurídica e normativa. Intuitivamente, identificou-se que o grau de Agressividade Ttributária mensura o ímpeto do sujeito passivo em reduzir o seu ônus fiscal em tributos explícitos, sem fazer qualquer restrição quanto à legalidade dos procedimentos às normas vigentes. 
Entre as perguntas de pesquisa que se revisaram ao longo do survey, sempre em uma perspectiva internacional e brasileira, destaquem-se a identificação de quais seriam os determinantes da Agressividade Tributária na firma, apreciando (i) características das firmas; (ii) atributos ambientais; (iii) restrições dos gatekeepers; e (iv) incentivos da firma. Revisaram-se igualmente os determinantes da agressividade fiscal dos gestores, identificando particularidades e incentivos específicos dos gestores que influenciam na Agressividade Tributária da firma.

Ainda na revisão da literatura, foi apreciada a influência da governança corporativa e da estrutura de propriedade e controle das empresas na sua Agressividade Tributária e quais as potenciais consequências econômico-financeiras da Agressividade Tributária para as firmas. Discutiu-se igualmente sobre as proxies empíricas existentes para mensurar a Agressividade Tributária.

O Brasil, com as alterações relativamente recentes na sua legislação contábil tributária, especificamente no tocante ao grau de conformidade dos lucros contábeis aos lucros tributários, oferece uma excelente plataforma para investigações mais aprofundadas em torno dessas temáticas, sendo muito útil tanto internamente como no exterior.

Como parte final da pesquisa, apresentam-se algumas oportunidades de pesquisa na temática, particularmente para a realidade brasileira. Da vinculação da Agressividade Tributária com o risco fiscal a sua relação com as escolhas contábeis, transitando por aprofundar os estudos das consequências e repensando as métricas de agressividade.

Em última ponderação, esperançoso, almeja-se que este survey se constitua em semente para futuras pesquisas nesta temática contábil tão instigante, atual e relevante da Agressividade Tributária.

\section{Referências}

Allen, A., Francis, B. B., Wu, Q. \& Zhao, Y. (2016). Analyst coverage and corporate tax aggressiveness. Journal of Banking \& Finance, 73(Supplement C), pp. 84-98. doi: https://doi.org/10.1016/j.jbankfin.2016.09.004

Araújo, R. A. de M. \& Leite Filho, P. A. M. (2017). Análise da Relação entre o Nível de Agressividade Fiscal e a Rentabilidade das Empresas da BMßFBovespa e NYSE. Anais do Congresso Anpcont, Belo Horizonte, MG, Brasil, 11.

Armstrong, C. S., Blouin, J. L., Jagolinzer, A. D. \& Larcker, D. F. (2015). Corporate governance, incentives, and tax avoidance. Journal of Accounting and Economics, 60(1), pp. 1-17. doi: https://doi.org/10.1016/j.jacceco.2015.02.003

Armstrong, C. S., Blouin, J. L., \& Larcker, D. F. (2012). The incentives for tax planning. Journal of Accounting and Economics, 53(1), pp. 391-411. doi: https://doi.org/10.1016/j.jacceco.2011.04.001

Austin, C. R., \& Wilson, R. J. (2017). An Examination of Reputational Costs and Tax Avoidance: Evidence from Firms with Valuable Consumer Brands. Journal of the American Taxation Association, 39(1), pp. 67-93. doi: https://doi.org/10.2308/atax-51634

Badertscher, B. A., Katz, S. P., \& Rego, S. O. (2013). The separation of ownership and control and corporate tax avoidance. Journal of Accounting and Economics, 56(2), pp. 228-250. doi: https://doi.org/10.1016/j.jacceco.2013.08.005

Bauer, A. M. (2016). Tax Avoidance and the Implications of Weak Internal Controls. Contemporary Accounting Research, 33(2), pp. 449-486. doi: https://doi.org/10.1111/1911-3846.12151

Beck, P. J., \& Lisowsky, P. (2014). Tax Uncertainty and Voluntary Real-Time Tax Audits. The Accounting Review, 89(3), pp. 867-901. doi: https://doi.org/10.2308/accr-50677

Beng Wee Goh, Jimmy Lee, Chee Yeow Lim, \& Shevlin, T. (2016). The Effect of Corporate Tax Avoidance on the Cost of Equity. The Accounting Review, 91(6), pp. 1647-1670. doi: https://doi.org/10.2308/accr-51432 
BEPS Actions - OECD. (2017). Recuperado em 6 de Novembro, 2017, de http://www.oecd.org/tax/beps/beps-actions.htm

Bertrand, M., \& Schoar, A. (2003). Managing with Style: The Effect of Managers on Firm Policies. The Quarterly Journal of Economics, 118(4), pp. 1169-1208. doi: https://doi.org/10.1162/003355303322552775

Bird, A., \& Karolyi, S. A. (2017). Governance and taxes: evidence from regression discontinuity. The Accounting Review, 92(1), pp. 29-50. doi: https://doi.org/ doi.org/10.2308/accr-51520

Bis, C., \& Martinez, A. L. (2017). Agressividade Fiscal em Empresas Brasileiras com Controle de Capital Estrangeiro. Anais da EnANPAD 2017, São Paulo, SP, Brasil, 41.

Blouin, J. (2014). Defining and Measuring Tax Planning Aggressiveness. National Tax Journal, 67(4), pp. 875900.

Boone, J. P., Khurana, I. K., \& Raman, K. K. (2013). Religiosity and Tax Avoidance. Journal of the American Taxation Association, 35(1), pp. 53-84. doi: https://doi.org/10.2308/atax-50341

Bozanic, Z., Hoopes, J. L., Thornock, J. R., \& Williams, B. M. (2017). IRS Attention. Journal of Accounting Research, 55(1), pp. 79-114. doi: https://doi.org/10.1111/1475-679X.12154

Brown, J. L. (2011). The Spread of Aggressive Corporate Tax Reporting: A Detailed Examination of the Corporate-Owned Life Insurance Shelter. The Accounting Review, 86(1), pp. 23-57. doi: https://doi.org/10.2308/ accr.00000008

Brown, J. L., \& Drake, K. D. (2013). Network Ties Among Low-Tax Firms. The Accounting Review, 89(2), pp. 483-510. doi: https://doi.org/10.2308/accr-50648

Brown, J. L., Drake, K., \& Wellman, L. (2015). The benefits of a relational approach to corporate political activity: evidence from political contributions to tax policymakers. Journal of the American Taxation Association, 37(1), pp. 69-102. doi: https://doi.org/10.2308/atax-50908

Brunozi Junior, A. C. (2016). Os efeitos das book-tax differences anormais na qualidade dos resultados contábeis em empresas de capital aberto listadas no Brasil. Tese de Doutoramento em Ciências Contábeis, Universidade do Vale do Rio dos Sinos(Unisinos), São Leopoldo, RS, Brasil.

Cabello, O. G., \& Gaio, L. E. (2017). Tax Avoidance in Management-Owned Firms: Evidence from Brazil. Anais da EnANPAD, São Paulo, SP, Brasil, 41.

Carvalho, V. G. (2017). Influência do Tax Management e Tax Aggressiveness na Qualidade da Previsão do Lucro por Ação pelos Analistas. Anais da EnANPAD, São Paulo, SP. Brasil, 41.

Chan, K. H., Phyllis Lai Lan Mo, \& Tanya Tang. (2016). Tax Avoidance and Tunneling: Empirical Analysis from an Agency Perspective. Journal of International Accounting Research, 15(3), pp. 49-66. doi: https://doi. org/10.2308/jiar-51345

Chaudhry, N., Au Yong, H. H., \& Veld, C. (2017). Tax avoidance in response to a decline in the funding status of defined benefit pension plans. Journal of International Financial Markets, Institutions and Money, 48(Supplement C), pp. 99-116. doi: https://doi.org/10.1016/j.intfin.2016.12.004

Chen, S., Chen, X., Cheng, Q., \& Shevlin, T. (2010). Are family firms more tax aggressive than non-family firms? Journal of Financial Economics, 95(1), pp. 41-61. doi: https://doi.org/10.1016/j.jfineco.2009.02.003

Cheng, C. S. A., Huang, H. H., Yinghua Li, \& Stanfield, J. (2012). The Effect of Hedge Fund Activism on Corporate Tax Avoidance. The Accounting Review, 87(5), pp. 1493-1526. doi: https://doi.org/10.2308/accr-50195

Chi, S., Huang, S. X., \& Sanchez, J. M. (2017). CEO Inside Debt Incentives and Corporate Tax Sheltering. Journal of Accounting Research, 55(4), pp. 837-876. doi: https://doi.org/10.1111/1475-679X.12169

Chyz, J. A. (2013). Personally tax aggressive executives and corporate tax sheltering. Journal of Accounting and Economics, 56(2), pp. 311-328. doi: https://doi.org/10.1016/j.jacceco.2013.09.003 
Chyz, J. A., Ching Leung, W. S., Zhen Li, O., \& Meng Rui, O. (2013). Labor unions and tax aggressiveness. Journal of Financial Economics, 108(3), pp. 675-698. doi: https://doi.org/10.1016/j.jineco.2013.01.012

Chyz, J. A., \& Gaertner, F. B. (2017). Can Paying «Too Much» or «Too Little» Tax Contribute to Forced CEO Turnover? The Accounting Review. doi: https://doi.org/10.2308/accr-51767

Cook, K. A., Moser, W. J., \& Omer, T. C. (2017). Tax avoidance and ex ante cost of capital. Journal of Business Finance \& Accounting, 44(7-8), pp. 1109-1136. doi: https://doi.org/10.1111/jbfa.12258

Da Silva, R., \& Martinez, A. L. (2017). Restrição Financeira e Agressividade Fiscal nas Empresas Brasileiras de Capital Aberto. Anais do Congresso Anpcont, Belo Horizonte, MG, Brasil, 11.

Davis, A. K., Guenther, D. A., Krull, L. K., \& Williams, B. M. (2016). Do socially responsible firms pay more taxes? The Accounting Review, 91(1), pp. 47-68. doi: https://doi.org/10.2308/accr-51224

De Simone, L. (2016). Does a common set of accounting standards affect tax-motivated income shifting for multinational firms? Journal of Accounting and Economics, 61(1), pp. 145-165. doi: https://doi.org/10.1016/j. jacceco.2015.06.002

De Simone, L., Ege, M. S., \& Stomberg, B. (2015). Internal control quality: the role of auditor-provided tax services. The Accounting Review, 90(4), pp. 1469-1496. doi: https://doi.org/10.2308/accr-50975

De Simone, L., Klassen, K. J., \& Seidman, J. K. (2017). Unprofitable affiliates and income shifting behavior. The Accounting Review, 92(3), pp. 113-136. doi: https://doi.org/10.2308/accr-51555

De Simone, L., Sansing, R. C., \& Seidman, J. K. (2013). When are enhanced relationship tax compliance programs mutually beneficial? The Accounting Review, 88(6), pp. 1971-1991. doi: https://doi.org/10.2308/accr-50525

Desai, M. A., \& Dharmapala, D. (2006). Corporate tax avoidance and high-powered incentives. Journal of Financial Economics, 79(1), pp. 145-179. doi: https://doi.org/10.1016/j.jfineco.2005.02.002

Desai, M. A., \& Dharmapala, D. (2009). Corporate Tax Avoidance and Firm Value. Review of Economics and Statistics, 91(3), pp. 537-546. doi: https://doi.org/10.1162/rest.91.3.537

Donohoe, M. P. (2015). The economic effects of financial derivatives on corporate tax avoidance. Journal of Accounting and Economics, 59(1), pp. 1-24. doi: https://doi.org/10.1016/j.jacceco.2014.11.001

Dourado, A. P. (2017). Part 1: Tax Avoidance Revisited in the EU BEPS Context. Em Tax Avoidance Revisited in the EU BEPS Context: 2016 eatlp congress munich, 2-4 june 2016. (pp. 199-232). Amsterdam: Intl Bureau of Fiscal Doc. Holanda.

Dyreng, S. D., Hanlon, M., \& Maydew, E. L. (2008). Long-Run Corporate Tax Avoidance. The Accounting Review, 83(1), pp 61-82. doi: https://doi.org/10.2308/accr.2008.83.1.61

Dyreng, S. D., Hanlon, M., Maydew, E. L., \& Thornock, J. R. (2017). Changes in corporate effective tax rates over the past 25 years. Journal of Financial Economics, 124(3), pp. 441-463. doi: https://doi.org/10.1016/j. jineco.2017.04.001

Dyreng, S. D., Hoopes, J. L., \& Wilde, J. H. (2016). Public Pressure and Corporate Tax Behavior. Journal of Accounting Research, 54(1), pp. 147-186. doi: https://doi.org/10.1111/1475-679X.12101

Dyreng, S. D., \& Lindsey, B. P. (2009). Using Financial Accounting Data to Examine the Effect of Foreign Operations Located in Tax Havens and Other Countries on U.S. Multinational Firms' Tax Rates. Journal of Accounting Research, 47(5), pp. 1283-1316. doi: https://doi.org/10.1111/j.1475-679X.2009.00346.x

Dyreng, S. D., Lindsey, B. P., Markle, K. S., \& Shackelford, D. A. (2015). The effect of tax and nontax country characteristics on the global equity supply chains of U.S. multinationals. Journal of Accounting and Economics, 59(2), pp. 182-202. doi: https://doi.org/10.1016/j.jacceco.2015.01.003

Dyreng, S. D., Mayew, W. J., \& Williams, C. D. (2012). Religious Social Norms and Corporate Financial Reporting. Journal of Business Finance \& Accounting, 39(7/8), pp. 845-875. doi: https://doi.org/10.1111/j.14685957.2012.02295.x 
Edwards, A., Schwab, C., \& Shevlin, T. (2016). Financial constraints and cash tax savings. The Accounting Review, 91(3), pp. 859-881. doi: https://doi.org/10.2308/accr-51282

Fernandes, V. L., Martinez, A. L., \& Nossa, V. (2013). The Influence of the Best Corporate Governance Practices on the Allocation of Value Added to Taxes. A Brazilian Case. Contabilidade, Gestão e Governança, 16(3), pp. 58-69.

Ferreira, F. R., Martinez, A. L., Costa, F. M. da, \& Passamani, R. R. (2012). Book-tax differences e gerenciamento de resultados no mercado de ações do Brasil. Revista de Administração de Empresas, 52(5), pp. 488-501. doi: https://doi.org/10.1590/S0034-75902012000500002

Fonseca, N. M., \& Martinez, A. L. (2017). A influência da Estrutura de Controle na Agressividade Fiscal. Anais da EnANPAD, São Paulo, SP, Brasil, 41.

França, C. J. de, Moraes, A. M. L. de M. de, \& Martinez, A. L. (2015). Tributação Implícita e Clientelas, Arbitragem, Restrições e Fricções. Revista de Administração e Contabilidade da FAT, 7(1), pp. 5-18.

Francis, B. B., Hasan, I., Qiang Wu, \& Meng Yan. (2014). Are Female CFOs Less Tax Aggressive? Evidence from Tax Aggressiveness. Journal of the American Taxation Association, 36(2), pp. 171-202. doi: https://doi. org/10.2308/atax-50819

Frank, M. M., Lynch, L. J., \& Rego, S. O. (2009). Tax Reporting Aggressiveness and Its Relation to Aggressive Financial Reporting. The Accounting Review, 84(2), pp. 467-496. doi: https://doi.org/10.2308 / accr.2009.84.2.467

Gallemore, J., \& Labro, E. (2015). The importance of the internal information environment for tax avoidance. Journal of Accounting and Economics, 60(1), pp. 149-167. doi: https://doi.org/10.1016/j.jacceco.2014.09.005

Gomes, A. P. M. (2016). Corporate Governance Characteristics as a Stimulus to Tax Management. Revista Contabilidade \& Finanças, 27(71), pp. 149-168. doi: https://doi.org/10.1590/1808-057x201500750

Graham, J. R., Hanlon, M., Shevlin, T., \& Shroff, N. (2014). Incentives for Tax Planning and Avoidance: Evidence from the Field. The Accounting Review, 89(3), pp. 991-1023. doi: https://doi.org/10.2308/accr-50678

Guenther, D. A., Matsunaga, S. R., \& Williams, B. M. (2017). Is Tax Avoidance Related to Firm Risk? Accounting Review, 92(1), pp. 115-136. doi: https://doi.org/10.2308/accr-51408

Guimarães, G. O. M., Macedo, M. A. da S., \& Cruz, C. F. da. (2016). Análise da Alíquota Efetiva de Tributos Sobre o Lucro no Brasil: Um Estudo com foco na ETRt e na ETRc. Enfoque: Reflexão Contábil, 35(1), pp. 1-16. doi: https://doi.org/10.4025/enfoque.v35i1.30570

Hanlon, M., \& Heitzman, S. (2010). A review of tax research. Journal of Accounting and Economics, 50(2), pp. 127-178. doi: https://doi.org/10.1016/j.jacceco.2010.09.002

Henry, E., Massel, N., \& Towery, E. (2016). Increased Tax Disclosures and Corporate Tax Avoidance. National Tax Journal, 69(4), pp. 809-829. doi: https://doi.org/10.17310/ntj.2016.4.04

Higgins, D., Omer, T. C., \& Phillips, J. D. (2015). The Influence of a Firm's Business Strategy on its Tax Aggressiveness. Contemporary Accounting Research, 32(2), pp. 674-702. doi: https://doi.org/10.1111/19113846.12087

Hoi, C. K., Wu, Q., \& Zhang, H. (2013). Is Corporate Social Responsibility (CSR) Associated with Tax Avoidance? Evidence from Irresponsible CSR Activities. The Accounting Review, 88(6), pp. 2025-2059. doi: https://doi.org/10.2308/accr-50544

Hoopes, J. L., Mescall, D., \& Pittman, J. A. (2012). Do IRS Audits Deter Corporate Tax Avoidance? The Accounting Review, 87(5), pp. 1603-1639. doi: https://doi.org/10.2308/accr-50187

Hope, O.-K., Ma, M. (Shuai), \& Thomas, W. B. (2013). Tax avoidance and geographic earnings disclosure. Journal of Accounting and Economics, 56, pp. 170-189. doi: https://doi.org/10.1016/j.jacceco.2013.06.001 
Huang, H. H., Lobo, G. J., Wang, C., \& Xie, H. (2016). Customer concentration and corporate tax avoidance. Journal of Banking and Finance, 72, pp. 184-200. doi: https://doi.org/10.1016/j.jbankfin.2016.07.018

Huseynov, F., \& Klamm, B. K. (2012). Tax avoidance, tax management and corporate social responsibility. Journal of Corporate Finance, 18(4), pp. 804-827. doi: https://doi.org/10.1016/j.jcorpfin.2012.06.005

Jones, S., Baker, M., \& Lay, B. F. (2017). The relationship between CSR and tax avoidance: an international perspective. Australian Tax Forum, 32(1), pp. 95-127.

Khan, M., Srinivasan, S., \& Tan, L. (2017). Institutional Ownership and Corporate Tax Avoidance: New Evidence. The Accounting Review, 92(2), pp. 101-122. doi: https://doi.org/10.2308/accr-51529

Khurana, I. K., \& Moser, W. J. (2013). Institutional Shareholders' Investment Horizons and Tax Avoidance. Journal of the American Taxation Association, 35(1), pp 111-134.doi: https://doi.org/10.2308/atax-50315

Klassen, K. J., Lisowsky, P., \& Mescall, D. (2016). The Role of Auditors, Non-Auditors, and Internal Tax Departments in Corporate Tax Aggressiveness. The Accounting Review, 91(1), pp 179-205. doi: https://doi. org/10.2308/accr-51137

Koester, A., Shevlin, T., \& Wangerin, D. (2017). The Role of Managerial Ability in Corporate Tax Avoidance. Management Science, 63(10), pp 3285-3310. doi: https://doi.org/10.1287/mnsc.2016.2510

Kubick, T. R., \& Lockhart, G. B. (2017). Corporate tax aggressiveness and the maturity structure of debt. Advances in Accounting, 36(Supplement C), pp 50-57. doi: https://doi.org/10.1016/j.adiac.2016.10.001

Kubick, T. R., Lockhart, G. B., Mills, L. F., \& Robinson, J. R. (2017). IRS and corporate taxpayer effects of geographic proximity. Journal of Accounting and Economics, 63(2), pp. 428-453. doi: https://doi.org/10.1016/j. jacceco.2016.09.005

Kubick, T. R., Lynch, D. P., Mayberry, M. A., \& Omer, T. C. (2015). Product Market Power and Tax Avoidance: Market Leaders, Mimicking Strategies, and Stock Returns. Accounting Review, 90(2), pp. 675-702. doi: https://doi.org/10.2308/accr-50883

Kubick, T. R., \& Masli, A. N. S. (2016). Firm-level tournament incentives and corporate tax aggressiveness. Journal of Accounting and Public Policy, 35(1), pp. 66-83. doi: https://doi.org/10.1016/j.jaccpubpol.2015.08.002

Laguir, I., Staglianò, R., \& Elbaz, J. (2015). Does corporate social responsibility affect corporate tax aggressiveness? Journal of Cleaner Production, 107(Supplement C), pp. 662-675. doi: https://doi.org/10.1016/j. jclepro.2015.05.059

Lanis, R., Richardson, G., \& Taylor, G. (2017). Board of Director Gender and Corporate Tax Aggressiveness: An Empirical Analysis. Journal of Business Ethics, 144(3), pp. 577-596. doi: https://doi.org/10.1007/ s10551-015-2815-x

Law, K. K. F., \& Mills, L. F. (2015). Taxes and Financial Constraints: Evidence from Linguistic Cues. Journal of Accounting Research, 53(4), pp. 777-819. doi: https://doi.org/10.1111/1475-679X.12081

Law, K. K. F., \& Mills, L. F. (2017). Military experience and corporate tax avoidance. Review of Accounting Studies, 22(1), pp. 141-184. doi: https://doi.org/10.1007/s11142-016-9373-Z

Lee, N. (2017). Can Territorial Tax Compliance Systems Reduce the Tax Avoidance of Firms with Operations in Tax Havens? Emerging Markets Finance \& Trade, 53(4), pp. 968-985. doi: https://doi.org/10.1080/15 40496X.2016.1247690

Lennox, C., Lisowsky, P., \& Pittman, J. (2013). Tax Aggressiveness and Accounting Fraud. Journal of Accounting Research, 51(4), pp. 739-778. doi: https://doi.org/10.1111/joar.12002

Lietz, G. (2013). Tax Avoidance vs. Tax Aggressiveness: A Unifying Conceptual Framework (SSRN Scholarly Paper No. ID 2363828). Rochester, NY: Social Science Research Network. Obtido de doi: https://papers. ssrn.com/abstract=2363828 
Lisowsky, P. (2010). Seeking Shelter: Empirically Modeling Tax Shelters Using Financial Statement Information. The Accounting Review, 85(5), pp. 1693-1720. doi: https://doi.org/10.2308/accr.2010.85.5.1693

Markle, K. (2016). A Comparison of the Tax-Motivated Income Shifting of Multinationals in Territorial and Worldwide Countries. Contemporary Accounting Research, 33(1), pp. 7-43. doi: https://doi. org/10.1111/1911-3846.12148

Martinez, A. L. (2014). Recompensas positivas como mecanismo de incentivo ao compliance tributário. Caderno de Finanças Públicas / Escola de Administração Fazendária, 14, pp. 327-342.

Martinez, A. L., \& Coelho, L. F. D. A. (2016). Planejamento tributário com operações societárias: critérios de validade utilizados pelo CARF. Revista Contemporânea de Contabilidade, 13(30), pp. 193-213. doi: https://doi.org/10.5007/2175-8069.2016v13n30p193

Martinez, A. L., \& Dalfior, M. D. (2016). Agressividade Fiscal entre Companhias Controladoras e Controladas. Revista da Receita Federal: estudos tributários e aduaneiros, 2(1), pp. 344-362.

Martinez, A. L., Lessa, R. C., \& Moraes, A. de J. (2014). Remuneração dos auditores perante a agressividade tributaria e governança corporativa no Brasil. Revista Contabilidade e Controladoria, 6(3), pp. 8-18. doi: https://doi.org/10.5380/rcc.v6i3.34593

Martinez, A. L., \& Martins, V. A. M. (2016). Alavancagem Financeira e Agressividade Fiscal no Brasil. Revista de Contabilidade da UFBA, 10(3), pp. 4-22. doi: https://doi.org/10.9771/rc-ufba.v10i3.18383

Martinez, A. L., \& Ramalho, G. C. (2014). Family Firms and Tax Aggressiveness in Brazil. International Business Research, 7(3), pp. 129-136. doi: https://doi.org/10.5539/ibr.v7n3p129

Martinez, A. L., Ribeiro, A. C., \& Funchal, B. (2015). The Sarbanes Oxley Act and Taxation: A Study of the Effects on the Tax Aggressiveness of Brazilian Firms. Anais do Congresso da USP Controladoria e Contabilidade, São Paulo, SP, Brasil, 15.

Martinez, A. L., \& Silva, R. F. (2017). Agressividade Fiscal e o Custo de Capital de Terceiros no Brasil. Revista de Gestão, Finanças e Contabilidade, 7(1), pp. 240-251. doi: https://doi.org/10.18028/2238-5320/rgfc. v7n1p240-251

McGuire, S. T., Omer, T. C., \& Wang, D. (2012). Tax Avoidance: Does Tax-Specific Industry Expertise Make a Difference? The Accounting Review, 87(3), pp. 975-1003. doi: https://doi.org/10.2308/accr-10215

McGuire, S. T., Omer, T. C., \& Wilde, J. H. (2014). Investment Opportunity Sets, Operating Uncertainty, and Capital Market Pressure: Determinants of Investments in Tax Shelter Activities? Journal of the American Taxation Association, 36(1), pp. 1-26. doi: https://doi.org/10.2308/atax-50599

Medeiros, R. D. M., \& Costa, F. M. (2017). Extinção da Dedutibilidade dos Juros sobre o Capital Próprio: análise das propostas fundamentadas em capacidade contributivo e agressividade fiscal. Anais do Congresso Anpcont, Belo Horizonte, MG, Brasil, 11.

Miiller, D., \& Martinez, A. L. (2016). Book-Tax Difference, Earnings Management And Bonds Ratings in the Brazilian Market. Revista Universo Contábil, pp. 91-109. doi: https://doi.org/10.4270/ruc.2016323

Mills, L. F., Nutter, S. E., \& Schwab, C. M. (2013). The Effect of Political Sensitivity and Bargaining Power on Taxes: Evidence from Federal Contractors. The Accounting Review, 88(3), pp. 977-1005. doi: https://doi. org/10.2308/accr-50368

Motta, F., \& Martinez, A. (2015). Agressividade Fiscal em Sociedades de Economia Mista. Anais da Enanpad, Belo Horizonte,MG, Brasil, 39.

Olsen, K. J., \& Stekelberg, J. (2016). CEO Narcissism and Corporate Tax Sheltering. The Journal of the American Taxation Association, 38(1), pp. 1-22. doi: https://doi.org/10.2308/atax-51251 
Paste Junior, E. (2017). Eficiência das empresas e agressividade tributaria: Evidencias do Brasil, Dissertação de Mestrado em Ciências Contábeis, Fucape Business School - ES, Vitoria. Recuperado em 02 de novembro, 2017, de http://www.fucape.br/_public/producao_cientifica/8/Dissertação\%20-\%20Eloy\%20 Paste\%20Junior.pdf

Potin, S., Silva, V. C., Reina, D., \& Sarlo Neto, A. (2016). Análise da Relação de Dependência entre Proxies de Governança Corporativa, Planejamento Tributário e Retorno sobre Ativos das Empresas da BM\&FBOVESPA. Revista Organizações em Contexto, 12(23), pp. 455-478. doi: https://doi. org/10.15603/1982-8756/roc.v12n23p455-478

Ramos, M. C. (2017). Relação entre a agressividade tributária e refazimento das demonstrações financeiras: Evidências nas empresas brasileiras listadas na BMßFBovespa, Dissertação de Mestrado em Ciências Contábeis, Fucape Business School - RJ, Rio de Janeiro. Recuperado em 02 de novembro, 2017, de http://www. fucape.br/_public/producao_cientifica/18/Dissertaçao-Marcia\%20Correa\%20Ramos.pdf

Reinders, A. P. G. S., \& Martinez, A. L. (2016). Qual o efeito da Agressividade Tributária na Rentabilidade Futura? Uma análise das companhias abertas brasileiras. Anais do Congresso Anpcont, Ribeirão Preto, SP, Brasil, 10.

Richardson, G., Taylor, G., \& Lanis, R. (2015). The impact of financial distress on corporate tax avoidance spanning the global financial crisis: Evidence from Australia. Economic Modelling, 44(Supplement C), pp. 44-53. doi: https://doi.org/10.1016/j.econmod.2014.09.015

Richardson, G., Wang, B., \& Zhang, X. (2016). Ownership structure and corporate tax avoidance: Evidence from publicly listed private firms in China. Journal of Contemporary Accounting \& Economics, 12(2), pp. 141-158. doi: https://doi.org/10.1016/j.jcae.2016.06.003

Rodrigues, M. A. (2017). Demora na publicação das demonstrações contábeis e financeiras e a agressividade fiscal, Dissertação de Mestrado em Ciências Contábeis, Fucape Business School - RJ, Rio de Janeiro. Recuperado em 02 de novembro, 2017, de http://www.fucape.br/_public/producao_cientifica/18/Dissertaçao\%20-\%20Marluci\%20Azevedo\%20Rodrigues.pdf

Salihu, I. A., Annuar, H. A., \& Sheikh Obid, S. N. (2015). Foreign investors' interests and corporate tax avoidance: Evidence from an emerging economy. Journal of Contemporary Accounting \& Economics, 11(2), pp. 138-147. doi: https://doi.org/10.1016/j.jcae.2015.03.001

Santana, S. L. L., \& Rezende, A. J. (2016). Corporate tax avoidance and firm value: evidence from Brazil. Revista Contemporânea de Contabilidade, 13(30), pp. 114-133. doi: https://doi.org/10.5007/2175$-8069.2016 \mathrm{v} 13 \mathrm{n} 30 \mathrm{p} 114$

Scholes, M. S., Wolfson, M. A., Erickson, M. M., Hanlon, M. L., Maydew, E. L., \& Shevlin, T. (2015). Taxes and business strategy: a planning approach (Fifth edition). Boston: Pearson.

Schoueri, L. ., \& Freitas, R. de (Eds.). (2010). Planejamento tributário e o "propósito negocial»: mapeamento de decisões do Conselho de Contribuintes de 2002 a 2008. São Paulo: Quartier Latin.

Schoueri, L. E., \& Galendi Júnior, R. A. (2017). Thematic Reports : Brazil. Em Tax Avoidance Revisited in the EU BEPS Context: 2016 eatlp congress munich, pp. 2-4 june 2016. (pp. 199-232). Amsterdam: Intl Bureau of Fiscal Doc.

Seidman, J. K., \& Stomberg, B. (2017). Equity Compensation and Tax Avoidance: Disentangling Managerial Incentives from Tax Benefits and Reexamining the Effect of Shareholder Rights. Journal of the American Taxation Association, 39(2), pp. 21-41. doi: https://doi.org/10.2308/atax-51755

Shackelford, D. A., \& Shevlin, T. (2001). Empirical tax research in accounting. Journal of Accounting and Economics, 31(1-3), pp. 321-387. doi: https://doi.org/10.1016/S0165-4101(01)00022-2 
Shevlin, T., Thornock, J., \& Williams, B. (2017). An examination of firms' responses to tax forgiveness. Review of Accounting Studies, 22(2), pp. 577-607. doi: https://doi.org/10.1007/s11142-017-9390-6

Silva, J. M. da, \& Rezende, A. J. (2017). A influência do ciclo de vida organizacional sobre o nível de planejamento tributário. Anais do Congresso Anpcont, Belo Horizonte, MG, Brasil, 11.

Silva, J. D. R., \& Martinez, A. L. (2017). Agressividade Fiscal de Empresas Brasileiras com Transações entre Partes Relacionadas no Exterior. Anais do Congresso Anpcont, Belo Horizonte, MG, Brasil, 11.

Sundvik, D. (2017). Book-tax conformity and earnings management in response to tax rate cuts. Journal of International Accounting, Auditing and Taxation, 28, pp. 31-42. doi: https://doi.org/10.1016/j.intaccaudtax.2016.12.003

Tang, T. Y. H. (2015). Does Book-Tax Conformity Deter Opportunistic Book and Tax Reporting? An International Analysis. European Accounting Review, 24(3), pp. 441-469. doi: https://doi.org/10.1080/0963818 0.2014 .932297

Taylor, G., \& Richardson, G. (2012). International Corporate Tax Avoidance Practices: Evidence from Australian Firms. The International Journal of Accounting, 47(4), pp. 469-496. doi: https://doi.org/10.1016/j. intacc.2012.10.004

Towery, E. M. (2017). Unintended Consequences of Linking Tax Return Disclosures to Financial Reporting for Income Taxes: Evidence from Schedule UTP. The Accounting Review, 92(5), pp. 201-226. doi: https:// doi.org/10.2308/accr-51660

Vale, J. P. S. do, \& Nakao, S. H. (2017). Unconditional conservatism in Brazilian public companies and tax neutrality. Revista Contabilidade \& Finanças, 28(74), pp. 197-212. doi: https://doi.org/10.1590/1808$-057 \times 201702450$

Vello, A., \& Martinez, A. L. (2014). Planejamento tributário eficiente: uma análise de sua relação com o risco de mercado. Revista Contemporânea de Contabilidade, 11(23), pp. 117. doi: https://doi.org/10.5007/2175$-8069.2014 \mathrm{v} 1 \ln 23 \mathrm{p} 117$

Watson, L. (2015). Corporate Social Responsibility, Tax Avoidance, and Earnings Performance. The Journal of the American Taxation Association, 37(2), pp. 1-21. doi: https://doi.org/10.2308/atax-51022

Wilde, J. H. (2017). The Deterrent Effect of Employee Whistleblowing on Firms' Financial Misreporting and Tax Aggressiveness. The Accounting Review, 92(5), pp. 247-280. doi: https://doi.org/10.2308/accr-51661

Wilde, J. H., \& Wilson, R. J. (2017). Perspectives on Corporate Tax Avoidance: Observations from the Past Decade. SSRN Scholarly Paper, Rochester, NY. Obtido de doi: https://papers.ssrn.com/abstract=2964053

Wilson, R. J. (2009). An Examination of Corporate Tax Shelter Participants. The Accounting Review, 84(3), pp. 969-999. doi: https://doi.org/10.2308/accr.2009.84.3.969

Zheng, S. (2017). Can corporate diversification induce more tax avoidance? Journal of Multinational Financial Management, 41(Supplement C), pp. 47-60. doi: https://doi.org/10.1016/j.mulfin.2017.05.008 